\title{
Atrial Fibrillation and Left Ventricle Dysfunction Relationship (New Concept)
}

\section{Vladimir Tilman*}

Department of Cardiology, The Sheba Medical Center, Rehabilitation Hospital, Ramat Gan, Israel

\begin{abstract}
Aim: Atrial Fibrillation (AF) is the most common cardiac arrhythmia. The numerous researches about the causes, the mechanism of its development and its links with other diseases have not given definite answers to these many questions. I propose a hypothesis which can explain the reason for the development of AF, its correlation with various diseases and the interpretations of the well- known facts and phenomena associated with this arrhythmia.

Methods and results: The hypothesis results from the comparison of the hydrodynamics of the sinus rhythm and atrial fibrillation. According to this hypothesis AF is not a disease, but the protective physiological mechanism. In the conditions of left ventricle dysfunction/heart failure, the termination of the atrial mechanical systole reduces hydrostatic pressure in the pulmonary veins and alveolar capillaries which then reduces danger of pulmonary congestion. The hypothesis allows to build the algorithms of the relations between AF and various diseases.

Conclusion: The hypothesis suggested that $A F$ is a physiological protective mechanism that is activated in the conditions of LV dysfunction.
\end{abstract}

Keywords: Atrial fibrillation; Sinus rhythm; Hyperthyroidism, Mitral stenosis; Hypertension

\section{Introduction}

Atrial fibrillation (AF) is the most common form of arrhythmia. Every year, the number of patients with this rhythm disturbance increases. It is called the epidemic of the century [1]. Despite a number of investigations of the causes and mechanisms of the development of the $\mathrm{AF}$, many questions remain unanswered.

\section{Common knowledge about AF includes}

1. AF is the fibrillation of the atrial myocardium with a frequency of 450-550 impulses per minute with the termination of the mechanical atrial systole [2].

2. The rate of heart contractions (i.e. a ventricle response) depend on the function of the atrial-ventricular node and may be more often tachyform (greater than 100 beats per minute), but may be also the normoform and bradyform. The AF is a supraventricular arrhythmia [3].

3. AF is directly related to left ventricle (LV) dysfunction/heart failure (HF). The prevalence of $\mathrm{AF}$ in patients with $\mathrm{HF}$ increases with the severity of the disease. In patients with mild HF approx. 5\% have AF. From $10 \%$ to $25 \%$ among patients with moderate HF have AF up to $50 \%$ in patients with severe heart failure have AF [4-6].

4. Accordingly, AF is linked to several diseases in which LV dysfunction the most often develops, including hypertension (more than $50 \%$ of AF cases), mitral stenosis ( $40 \%$ and more), hyperthyroidism (15\%) and so on [7-11].

5. $\mathrm{AF}$ is in direct correlation with age, when congestive heart failure is the most common state in hospital patients 65 years and older. Considering that the proportion of elderly individuals is growing, the prevalence of AF will increase 2-5 fold by the year 2050 by projected ATRIA study [12].

\section{Unanswered questions associated with AF}

1. There are a number of theories about the mechanism of AF (multifocal, re-entry and etc.), but localization of an anatomical or histological substrate as a source of AF has still not been found $[13,14]$. Satisfactory results on cessation of AF by ablation of the orifices of the pulmonary veins suggest that the source of AF may be situated in the region of the pulmonary veins and the ablation only "cuts" the spread of arrhythmia. Genetic research is in favor of this theory. The genes identified include a number of transcription factors, which have been implicated in pulmonary vein development, and the new series of atrial fibrillation-related genes were found $[15,16]$. Aberrant triggering at the genetic level, perhaps due to mutation, may explain the cause of AF without organic pathology of the heart-Lone AF.

2. A lot of studies have been performed to compare the ratecontrol versus rhythm-control strategy in patients with AF (AFFIRM, RACE, STAF, AF-CHF etc.). It would seem that a return to normal sinus rhythm in conditions of LV dysfunction/HF should significantly improve the patients' states, and accordingly, the rhythm-control should be preferred. However, it turned out that the rhythm-control does not have an advantage over the rate-control, and even actually showed a trend towards harm in the general population. Current research is still unable to answer why this is [17-19].

3. There is also no answer to the question why in some cases, when the AF is returned to the sinus rhythm instead of the expected improvement, pulmonary edema develops. The same complication occurs in some cases, when the pacemaker is implanted in AF (pacemaker syndrome) [20,21].

4. There is no adequate answer, why after successful treatment of AF with rhythm-control (by medicine, cardioversion or ablation) arrhythmia spontaneously returns in many cases [22,23].

*Corresponding author: Vladimir Tilman, Department of Cardiology, The Sheba Medical Center, Rehabilitation Hospital, Ramat Gan, Eilat st.33, Apt 4, Holon, Israel, Tel:9720547345525; E-mail: water5945@gmail.com

Received December 18, 2017; Accepted December 27, 2017; Published December 29, 2017

Citation: Tilman V (2017) Atrial Fibrillation and Left Ventricle Dysfunction Relationship (New Concept). J Cardiovasc Dis Diagn S2: 002. doi:10.4172/2329 9517.S2-002

Copyright: (C) 2017 Tilman V. This is an open-access article distributed under the terms of the Creative Commons Attribution License, which permits unrestricted use, distribution, and reproduction in any medium, provided the original author and source are credited. 


\section{Methods}

Analyzing all this information, it is necessary to conclude that $\mathrm{AF}$ is somehow associated with the functional state of the heart, and a direct correlation between $\mathrm{AF}$ and $\mathrm{LV}$ dysfunction/HF is not accidental. It seems strange and illogical that against the background of such a serious complication as LV dysfunction/HF is added one more complication$\mathrm{AF}$. The question arises as to whether the AF gives any benefit for the in the condition of the LV dysfunction/HF.

I have proposed a hypothesis that $\mathrm{AF}$ is not an additional complication to the already existing LV dysfunction, but a genetically programmed physiological protective mechanism that is activated at a certain level within the development of heart failure. The essence of the defensive mechanism of AF is as follows. The end diastolic pressure (EDP) is the algebraic sum of the left ventricle diastolic pressure (LVdp) and the left atrial (LA) systolic pressure (atrial kick pressure, AKp). $\mathrm{EDP}=\mathrm{LVdp}+\mathrm{AKp}$. Normal range of the left ventricle EDP is $8 \mathrm{~mm}$ $\mathrm{Hg}-12 \mathrm{~mm} \mathrm{Hg}, \mathrm{AKp}$ is $2 \mathrm{~mm} \mathrm{Hg}-6 \mathrm{~mm} \mathrm{Hg}$. At the end of the diastole when the mitral valve (MV) is still open, EDP immediately transmitted and equalized (according to hydrodynamic law) throughout the communicating system of the left ventricle, left atrium, pulmonary veins (PV), and alveolar capillaries (AC), as there are no obstacles to the spread of the EDP wave. It is known that in the conditions of the LV dysfunction (HF varying degrees), EDP increases due to failure of the left ventricle (i.e. LV diastolic pressure component increases).

EDP (incr) $=$ LVdp (incr) + AKp. This increased EDP at the end of diastole with opened MV transmits to the entire communicating system of the LA-PV-AC with the threat of exit of fluid through the alveolarcapillary membrane with the danger of the development of pulmonary congestion or edema.

According to the hypothesis, in the conditions of LV dysfunction/ $\mathrm{HF}$ when EDP increases to a certain critical value, the source of AF which is supposedly in the pulmonary veins, reacts to a dangerous increase in the pressure in the LA-PV-AC system and activates its mechanism. AF stops mechanical left atrial systole thus negating the component of systolic LA pressure (AKp) from the total pressure in the LA-PV-AC system. EDP=LVdp (without AKp). This leads to a reduction of pressure in the alveolar capillaries and reduces the threat of pulmonary congestion. The right atrium (RA) is also involved in AF. A termination of the mechanical systole of the RA leads to a reduction in EDP of the right ventricle (RV) with decreasing pressure of systemic venous inflow, resulting in a reduction of the preload. It means that $\mathrm{AF}$ is synergistic in the hydrodynamic effect, due to the termination of a mechanical systole in both the LA and RA. Thus, it can be assumed that in the conditions of the development of LV dysfunction/HF the AF plays the role in primary prevention of pulmonary congestion.

The "unanswered questions" above, through this interpretation, are therefore enlightened. The hypothesis explains:

1. Why the source of AF is preferably in the pulmonary veins (as it is a strategically important area).

2. Why rate-control is more preferable in the cases of the LV dysfunction/HF.

3. Why complications arise when the sinus rhythm is restored in the cases with increased pressure in the system of LA-PV-AC.

4. The reason for the tendency of the AF to return. According to this hypothesis the heart in the state of the LV dysfunction/HF "needs" AF, because it receives a more favorable hydrodynamic effect. Therefore, most of the symptoms attributed to AF are actually symptoms of a developing LV dysfunction.

As with any other compensatory mechanism, AF has its drawbacks. Firstly, the termination of the mechanical systole of the left atrium transforms the turbulent movement of the blood to laminar movement, as there is no alternation of pressure changes. Secondly, in the absence of the contraction of the myocardium of the atrium, blood can stagnate in the appendage potentially leading to thrombosis and possible thromboembolism to the brain $[24,25]$. Also, a very rapid ventricular response (although it is a function of AV node) can exacerbate HF.

\section{Results and Discussion}

Thus, the proposed hypothesis suggests that AF is a physiological protective mechanism, most likely genetically programmed, that decreases the pressure in the LA-PV-AC system by terminating the atrial mechanical systole. The hypothesis makes it possible to build an algorithm for the appearance of AF for various diseases.

\section{Hypertension}

Increased peripheral vascular resistance - increased systemic blood pressure - hypertrophy of LV with gradual development of LV dysfunction - increased EDP - increased pressure in the LA-PVAC system - danger of pulmonary congestion - activation of AF termination of atrial mechanical systole - reduction of the pressure in the LA-PV-AC system in the left side and reduction of the preload in the right side of the heart.

\section{Mitral stenosis}

Obstruction to LV inflow at a level of the mitral valve - increasing the resting diastolic mitral valve gradient - increasing pressure in the LA-PV-AC system - danger of pulmonary congestion - activation of AF - termination of atrial mechanical systole - reduction of the pressure in the LA-PV-AC system.

\section{Hyperthyroidism}

The hypermetabolic condition of thyrotoxicosis - prolonged tachycardia - tachycardia mediated cardiomyopathy - LV dysfunction with elevated EDP - increasing pressure in the LA-PV-AC system danger of pulmonary congestion - activation of AF - termination of atrial mechanical systole - reduction of pressure in the LA-PV-AC system. In the same way we can build an algorithm for the relationship between $\mathrm{AF}$ and other diseases, which lead to LV dysfunction

\section{Conclusion}

The above proposed hypothesis can suggest a scenario for the development of AF. Each individual has a protective physiological mechanism in the pulmonary veins, most likely genetically programmed. When the pressure in the LA-PV-AC system increases to a certain critical value in the conditions of $L V$ dysfunction/HF, the AF mechanism is activated. AF terminates mechanical systole of the LA excluding the component of systolic left atrial pressure from the total pressure in the LA-PV-AC system, and of the RA with effect of reducing preload, and thus decrease the threat of the development of pulmonary congestion or edema. This hypothesis may explain a lot of the known facts associated with $\mathrm{AF}$ and gives answers to many questions linked with this arrhythmia.

\section{References}

1. Chugh SS, Havmoeller R, Narayanan K, Singh D, Rienstra M, et al. (2014) Worldwide epidemiology of atrial fibrillation: A global burden of disease 2010 study. Circulation 129: 837-847. 
Citation: Tilman V (2017) Atrial Fibrillation and Left Ventricle Dysfunction Relationship (New Concept). J Cardiovasc Dis Diagn S2: 002. doi:10.4172/2329-9517.S2-002

Page 3 of 3

2. Hendriks JM, Crijns HJ, Tieleman RG, Vrijhoef HJ (2013) The atrial fibrillation knowledge scale: Development, validation and results. Int J Cardiol 168: 14221428.

3. Munger TM, Wu LQ, Shen WK (2014) Atrial fibrillation. J Biomed Res 28: 1-17.

4. Mamas MA, Caldwell JC, Chacko S, Garratt CJ, Fath-Ordoubadi F, et al. (2009) A meta-analysis of the prognostic significance of atrial fibrillation in chronic heart failure. Eur J Heart Fail 11: 676-683.

5. Maisel WH, Stevenson LW (2003) Atrial fibrillation in heart failure: Epidemiology, pathophysiology, and rationale for therapy. Am J Cardiol 91: 2-8.

6. Deedwania PC, Lardizabal JA (2010) Atrial fibrillation in heart failure: A comprehensive review. Am J Med 123: 198-204.

7. Manolis AJ, Rosei EA, Coca A, Cifkova R, Erdine SE, et al. (2012) Hypertension and atrial fibrillation: Diagnostic approach, prevention and treatment. Position paper of the Working Group 'Hypertension Arrhythmias and Thrombosis' of the European Society of Hypertension. J Hypertens 30: 239-252.

8. Odutayo A, Wong CX, Hsiao AJ, Hopewell S, Douglas AG, et al. (2016) Atria fibrillation and risks of cardiovascular disease, renal disease, and death: Systematic review and meta-analysis. BMJ 354: i4482.

9. Gelson E, Gatzoulis M, Johnson M (2007) Valvular heart disease. BMJ 335 1042-1045.

10. Frost $L$, Vestergaard $P$, Mosekilde $L$ (2004) Hyperthyroidism and risk of atria fibrillation flutter - A population-based study. Arch Intern Med 164: 1675-1678.

11. Auer J, Scheibner P, Mische T, Langsteger W, Eber O, et al. (2001) Subclinical hyperthyroidism as a risk factor for atrial fibrillation. Am Heart J 142: 838-842.

12. Go AS, Hylek EM, Phillips KA, Chang YC, Henault LE, et al. (2001) Prevalence of diagnosed atrial fibrillation in adults: National implications for rhythm management and stroke prevention: The AnTicoagulation and risk factors in atrial fibrillation (ATRIA) study. JAMA 285: 2370-2375.

13. Lin WS, Prakash VS, Tai CT, Hsieh MH, Tsai CF, et al. (2000) Pulmonary vein morphology in patients with paroxysmal AF initiated by ectopic beats originating from the pulmonary veins: Implications for catheter ablation. Circulation. 101: $1274-1281$.
14. PatelA Markowitz SM (2008)Atrial tachycardia: Mechanisms and management Expert Rev Cardiovasc Ther 6: 811-822.

15. Bedi M, McNamara D, London B, Schwartzman D (2006) Genetic susceptibility to atrial fibrillation in patients with congestive heart failure. Heart Rhythm 3 . 808-812.

16. Ellinor PT, MacRae CA (2003) The genetics of AF. J Cardiovasc Electrophysio 14: 1007-1009.

17. Gillis AM, Verma A, Talajic M, Nattel S, Dorian P (2011) Canadian Cardiovascular Society atrial fibrillation guidelines 2010: Rate and rhythm management. Can J Cardiol 27: 47-59.

18. Caldeira D, Cláudio D, Sampaio C (2012) Rate versus rhythm control in atrial fibrillation and clinical outcomes: Updated systematic review and meta-analysis of randomized controlled trials. Arch Cardiovasc Dis 105: 226-238.

19. Roy D, Talajic M, Nattel S, Wyse DG, Dorian P, et al. (2008) Rhythm control versus rate control for atrial fibrillation and heart failure. $\mathrm{N}$ Engl $\mathrm{J}$ Med 358 : 2667-2677.

20. Gowda RM, Misra D, Khan IA, Schweitzer P (2003) Acute pulmonary edema after successful cardioversion of atrial fibrillation. Am J Ther 10: 73-74.

21. Nagpal A, Baddour LM, Sohail MR (2010) Update on cardiovascular implantable electronic device infections and their management. A scientific statement from the American Heart Association. Circulation 121: 458-477.

22. Sotomi Y, Inoue K, Ito N, Kimura R, Toyoshima Y, et al. (2013) Incidence and risk factors for very late recurrence of atrial fibrillation after radiofrequency catheter ablation. Europace 15: 1581-1586.

23. Usui E, Miyazaki S, Taniguchi H, Ichihara N, Kanaji Y, et al. (2015) Recurrence after long-term success in catheter ablation of paroxysmal atrial fibrillation. Heart Rhythm 12: 893-898.

24. Tilman V (2016) Atrial fibrillation: In the light of new hypothesis. Med Hypotheses 86: $10-13$

25. Pálinkeás A, Antonielli E, Pizzuti A, Gruber N, Varga A, et al. (2002) Clinica value of left atrial appendage flow for prediction of long-term sinus rhythm maintenance in patients with nonvalvular atrial fibrillation. J Am Coll Cardiol 39: $1443-1449$. 